\title{
«Einige waren der Ansicht, man sollte das nicht öffentlich sagen»
}

Interview: Stéphane Praz

Wissenschaftsjournalist

«HIV-infizierte Menschen ohne andere STD sind unter wirksamer antiretroviraler Therapie sexuell nicht infektiös.» So lauteten Titel und zugleich Kernaussage eines Beitrags, den vier Infektiologen im Namen der Eidgenössischen Kommission für Aids-Fragen* im Januar 2008 in der SÄZ publizierten. Über die Landesgrenzen hinaus stiess das Swiss Statement auf helle Begeisterung ebenso wie auf harsche Kritik. Erstautor Pietro Vernazza spricht im Interview über Hintergründe und Folgen.

Herr Professor Vernazza, nach der Veröffentlichung des Swiss Statement wurden Sie und Ihre Kollegen teilweise mit harscher Kritik konfrontiert. Was warf man Ihnen vor?

Grundsätzlich gab es zwei Arten von Kritik. Einige zweifelten am Sachverhalt an sich. Andere fochten diesen nicht an, waren aber der Ansicht, man sollte nicht öffentlich sagen, dass HIV-Patienten unter wirksamer Therapie sexuell nicht infektiös sind.

\section{Weshalb sollte man das nicht sagen?}

Präventionskreise äusserten die Besorgnis, dass in der Öffentlichkeit aus "Mach's mit» ein «Mach's ohne» wird. Die Befürchtung hat sich jedoch nicht bestätigt. Das Swiss Statement richtete sich ohnehin nicht an eine breite Öffentlichkeit, sondern an Menschen mit HIV und an Fachleute.

Das Swiss Statement berührt die Prävention allerdings schon: Je mehr Menschen mit HIV unter Therapie sind, desto weniger können das Virus potentiell weitergeben.

Es ist sozusagen eine willkommene Nebenwirkung der Therapie, dass sie die weitere Übertragung von HIV sehr effizient verhindert. International, in besonders stark betroffenen Ländern, ist das von grosser Bedeutung, in der Schweiz eher nicht. Insofern ging es uns nicht um Präventionsaspekte. Aber klar, es ist sicherer, ohne Gummi mit einem Mann unter erfolgreicher HIV-Therapie zu schlafen, als mit einem, der seinen HIV-Status nicht kennt. Und aus individueller Sicht kann es ein Anreiz zur Therapie sein, wenn man mit der Therapie nicht mehr infektiös ist.
Welche Ziele verfolgten Sie mit dem Swiss Statement? Wir wollten HIV-Patienten ein normales Leben ermöglichen, ohne dass sie sich selbst als permanente Gefahr betrachten müssen. Und wir wollten auf die Rechtsprechung in der Schweiz einwirken. Diese beurteilte damals noch jeglichen ungeschützten Sexualverkehr von HIV-positiven Menschen als Straftat. Beide Ziele haben wir erreicht: Mit dem revidierten Epidemiengesetz werden Menschen mit HIV nicht mehr strafrechtlich verfolgt, wenn sie mit jemandem einvernehmlichen Sex ohne Kondom haben. Und eine Studie der Schweizerischen HIV-Kohorte hat gezeigt, dass das Swiss Statement das Sexualverhalten von HIV-Patienten beeinflusst. Oder mindestens bewirkte es, dass Patienten offener über ihr wahres Sexualverhalten berichten.

Sie meinen, Menschen mit HIV hatten schon früher einvernehmlichen Sex ohne Kondom, aber es durfte niemand wissen?

Dank dem Swiss Statement und seinen Folgen können sie mittlerweile auch darüber sprechen, ohne immer Angst vor Strafverfolgung haben zu müssen. Viele wurden zudem von der sehr belastenden - wenn auch unbegründeten - Angst befreit, sie könnten irgendwann einmal jemanden in einer ganz normalen Alltagssituation anstecken. Für diese Menschen war das Swiss Statement eine grosse Erleichterung. Weltweit zeigten sich Betroffenenorganisationen denn auch begeistert.

Obwohl zum Teil auch namhafte Fachleute der Ansicht waren, Ihre Aussagen seien nicht genügend 
erhärtet. Auf welcher wissenschaftlichen Evidenz basiert das Swiss Statement?

Das wichtigste Argument war: Man hat weltweit noch nie eine Übertragung beobachtet, die von einem HIVPatienten unter erfolgreicher Therapie ausgegangen ist. Zudem gab es auch schon einzelne Studien und die biologische Evidenz, wonach eine Übertragung als sehr unwahrscheinlich eingestuft werden musste. Eigentlich musste man sich umgekehrt fragen, welche Evidenz es gab, Menschen unter Therapie für infektiös zu halten. Diese Meinung hatte schliesslich einschneidende Konsequenzen für die Betroffenen.

Ihr Argument war also nicht, dass Sie Ihre Aussage beweisen konnten, sondern dass umgekehrt noch niemand das Gegenteil bewiesen hatte?

Damit hatten einige Kollegen zunächst Mühe, weil Sie sich gewohnt sind, dass insbesondere solch wichtige Erkenntnisse mit Studien belegt werden. Doch so aussergewöhnlich war unser Ansatz nicht. Die Evidenz, dass HIV beim Küssen nicht übertragen wird, beruht auf derselben Argumentation. Als man das 1987 publizierte, war die Datenlage sogar deutlich weniger robust.

Trotzdem brauchte es erst die Bestätigung durch eine grosse prospektive Studie, bis sich zum Beispiel auch die WHO den Aussagen des Swiss Statement anschloss. Die HPTN-52-Studie hat sicher sehr geholfen. Bereits die Zwischenresultate waren ja so deutlich, dass man die Studie abbrach. Allerdings muss man anfügen, dass das US-amerikanische CDC das Swiss Statement immer noch nicht akzeptiert hat, dafür in der Prävention die PrEP (präventive Einnahme von HIV-Medikamenten für HIV-negative Personen, Anm. d. Red.) promotet. Das ist inkonsequent, aber natürlich nützlich für die Pharmaindustrie. Und es legt den Verdacht nahe, dass das Swiss Statement nicht aufgrund fehlender Evidenz kritisiert wurde. In Diskussionen habe ich immer wieder Personen erlebt, die Menschen mit HIV gerne weiterhin Restriktionen auferlegt hätten. Etwa Hürden beim Kinderwunsch. Ich glaube, das hat viel mit Schuldzuweisung zu tun.

Auch zu einzelnen Punkten des Swiss Statement gibt es immer wieder Diskussionen. So zur Frage, ob die Nichtinfektiosität sowohl bei vaginalem wie bei analem Geschlechtsverkehr gilt. Denn an der angesprochenen HPTN-52-Studie nahmen ausschliesslich heterosexuelle Paare teil.

Wie gesagt, es wurde noch nie eine Übertragung durch einen HIV-Patienten unter erfolgreicher Therapie beobachtet. Auch nicht bei schwulen Männern.
Damit ein HIV-Patient als sexuell nicht infektiös gilt, muss seine Viruslast unter der Nachweisgrenze liegen, also unter fünfzig Viren pro Milliliter Blut. Es ist allerdings bekannt, dass dieser Wert kurzzeitig in die Höhe schnellen kann, wegen einer Grippe, wegen einer Impfung, teilweise auch ohne ersichtliche Gründe. Wie stabil ist «nicht infektiös»?

In den kontrollierten Studien genügte eine Therapie von mindestens drei Monaten Dauer, um die Viruslast stabil zu unterdrücken. Wir gehen davon aus, dass die kleinen Virusschwankungen bis zweihundert Kopien pro Milliliter Blut keinen relevanten Einfluss auf die Infektiosität haben.

\section{Bei welcher Viruslast wird es kritisch hinsichtlich infektiös oder nicht infektiös?}

Hierzu gibt es keine genauen Zahlen. Eine ältere Untersuchung hat infektiöses Virus im Sperma nur bei einer Viruslast von über tausend Kopien pro Milliliter im Blut nachweisen können. Diesen Wert fanden wir auch bei mathematischen Modellrechnungen. Doch bisher lässt sich das nicht ganz genau beziffern.

\section{Es gibt auch Faktoren, welche die Wirksamkeit} der Therapie längerfristig einschränken können, beispielsweise eine schlechte Adhärenz oder Wechselwirkungen mit anderen Medikamenten. Wie lassen sich diese kontrollieren?

$\mathrm{Zu}$ einer wirksamen Therapie gehört, dass die Adhärenz erprobt ist und die Patienten entsprechend sensibilisiert sind. Wenn eine Therapie über ein bis zwei Jahre gut funktioniert hat, passiert nichts Unvorhergesehenes, solange sie der Patient weiter einnimmt.

\section{Zusätzlich zu einer wirksamen Therapie darf keine} weitere sexuell übertragbare Infektion vorliegen, damit ein HIV-Patient als sexuell nicht infektiös gilt. Wie stark beeinflussen andere sexuell übertragbare Infektionen die Viruslast?

In Studien, auch in der HPTN-52, zeigt sich kein Einfluss von STI. Wir haben das Swiss Statement bewusst sehr konservativ formuliert. Die Einschränkung betreffend STI war nicht zwingend. Heute würden wir diesen Punkt wahrscheinlich weglassen.

\section{Sie würden das Swiss Statement heute anders formulieren?}

Ich denke ja. Aufgrund der neu gewonnenen Erkennt nisse darf man sagen: Wer unter funktionierender HIV-Therapie ist, ist nicht infektiös. Basta. 